\title{
A solid transportation problem with safety factor under different uncertainty environments
}

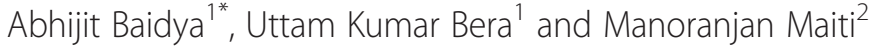

\author{
* Correspondence: \\ abhijitnita@yahoo.in \\ ${ }^{1}$ Department of Mathematics, \\ National Institute of Technology, \\ Agartala, Jirania 799055, West \\ Tripura, India \\ Full list of author information is \\ available at the end of the article
}

\begin{abstract}
In this paper, we introduce a new concept 'safety factor' in a transportation problem. When items are transported from plants to destinations through different conveyances, there are some difficulties/risks to transport the items due to bad road, insurgency, land slide, etc. in some routes. Due to these, a desired total safety factor is being introduced, and depending upon the nature of the safety factor, we develop five models. In this paper, a solid transportation problem (STP) with imprecise unit costs is considered. The sources' availabilities, destinations' demands, and capacities of conveyances are also represented by fuzzy numbers like trapezoidal and triangular numbers. The transportation problem has been formulated with and without a safety factor. To reduce the different models into its crisp equivalent, we introduce different methods as chance-constraint programming, an approach using interval approximation of fuzzy numbers and the application of the expected value model. Generalized reduced gradient technique is used to find the optimal solutions for a set of given numerical data. To illustrate the model, a numerical example has been presented and solved using LINGO.12 software. The effect of safety factors on transported amount is illustrated.
\end{abstract}

Keywords: Solid transportation problem; Safety factor; Fuzzy variable; Random variable; Hybrid variable; Expected value model; An approach using interval approximation of fuzzy number; Chance-constraint programming

\section{Introduction}

A transportation model plays a vital role in ensuring the efficient movement and intime availability of raw materials and finished goods from sources to destinations. Transportation problem is a linear programming problem that stemmed from a network structure consisting of a finite number of nodes and arcs attached to them. Effective algorithms have been developed to solve the transportation problem when the cost coefficients and the supply and demand quantities are known exactly. The occurrence of randomness and imprecision in the real world is inevitable owing to some unexpected situations. There are cases that the cost coefficients and the supply and demand quantities of a transportation problem may be uncertain due to some unmanageable factors. To deal quantitatively with imprecise information in making decisions, Bellman and Zadeh [1] and Zadeh [2,3] introduced the impression of fuzziness. In many industrial problems, a homogeneous product is delivered from an origin to a destination by means of different modes of transport called conveyances, such as

\section{Springer}


trucks, cargo flights, goods trains, and ships. As a generalization of traditional transportation problem, solid transportation problem (STP) was stated by Shell [4] in 1955, which he considered the three item properties in the constraint set instead of two items, namely source and destination. Haley [5] described a solution procedure of a solid transportation problem, which is an extension of the Modi method. In general, real-life problems are modeled with multi-objective functions which are measured in different respects, and they are noncommensurable and conflicting in nature. Furthermore, it is frequently difficult for the decision maker to combine the objective functions in one overall utility function. Due to insufficient information, lack of evidence, and fluctuating financial market, the available data of a transportation system such as resources, demands, and conveyance capacities are not always crisp or precise but are fuzzy, random, or both. Thus, the fuzziness and randomness can be present in the objective function as well as in the constraints of a STP. Since then, significant work has been done by researchers based on the uncertainty theory both in theoretical and practical aspects. If the cost parameters of the transportation problem are uncertain variables, we call the problem uncertain cost transportation problem. Along with global economic development, production and demand have much more importance. The importance of goods transportation is also increasingly reflected. Dealing with different types of uncertainty, Kundu et al. [6] solve solid transportation problems with budget constraint in an uncertain environment. Our aim is to formulate and solve solid transportation problems with safety constraints with different types of uncertain (fuzzy, random, and hybrid) parameters. Recently, Baidya et al. $[7,8]$ solved two problems based on safety factors and uncertainty in transportation problem.

In this paper, we develop five models and consider three types of uncertainty (stochastic, fuzzy, and hybrid) in different models for unit transportation cost and safety factor. Also, we consider resources, demands, and conveyance capacities as fuzzy. Model 1 was solved without considering a safety factor, but other models were solved with safety factors that are crisp, random, fuzzy, and hybrid for their respective models. To derive the crisp equivalences of the models, appropriate methods, i.e., chance-constrained programming, an approach that uses interval approximation of fuzzy numbers, and expected value model techniques are applied in these five different models. The models are illustrated with specific numerical data. Finally, all the models are solved using generalized reduced gradient method using LINGO.12 software. A comparison of the different models is presented.

\section{Literature review}

Zadeh [2] first introduced the concept of fuzzy set theory. Later on, several authors such as Zadeh [3], Kaufmann [9], Zimmermann [10], Liu [11], and Dubois and Prade [12] developed and applied the fuzzy set theory. Chanas and Kuchta [13] studied transportation problem with fuzzy cost coefficients. Jimenez and Verdegay [14] considered two types of uncertain STP, one with interval numbers and the other with fuzzy numbers. Charnes and Cooper [15] introduced chance-constrained programming to deal with uncertain environment. Liu and Iwamura [16] presented chanceconstrained programming with fuzzy parameters. Liu and Liu [17] presented expected value model for fuzzy programming. Yang and Liu [18] applied expected value model, chance-constrained programming model, and dependent-chance programming in a 
fixed charge solid transportation problem in a fuzzy environment. Mula, Poler, and Garcia Sabater [19] applied possibilistic programming approach to a material requirement planning problem with fuzzy constraints and fuzzy coefficients using the definition of possibility measure of fuzzy number. Chen and Tsai [20] considered time-cost trade-off problem with fuzzy parameters and constructed a two-level mathematical program for it using the concept $\alpha$-cut of fuzzy number. Fegad, Jadhav, and Muley [21] obtained an optimal solution of a transportation problem using interval and triangular membership functions. Hulsurkar, Biswal, and Sinha [22] applied the fuzzy programming approach to multi-objective stochastic programming problems after transforming them into crisp forms. Some methodologies to deal with objective function having random parameters are highlighted in Rao's book [23].

\section{Preliminaries}

Definition 1. (Fuzzy number) [24] A fuzzy subset $\tilde{A}$ of real number $\Re$ with membership function $\mu_{\tilde{A}}: \mathfrak{R} \rightarrow[0,1]$ is said to be a fuzzy number if the following conditions are met:

1. $\mu_{\tilde{A}}(x)$ is an upper semicontinuous membership function;

2. $\tilde{A}$ is normal, i.e., $\exists$ an element $x_{0}$ s.t. $\mu_{\tilde{A}}\left(x_{0}\right)=1$;

3. $\tilde{A}$ is fuzzy convex, i.e., $\mu_{\tilde{A}}\left(\lambda x_{1}+(1-\lambda) x_{2}\right) \geq \mu_{\tilde{A}}\left(x_{1}\right) \wedge \mu_{\tilde{A}}\left(x_{2}\right) \forall x_{1}, x_{2} \in \Re$ and $\lambda \in[0,1]$;

4. Support of $\tilde{A}=\left\{x \in \Re: \mu_{\tilde{A}}(x)>0\right\}$ is bounded.

Definition 2. (Triangular fuzzy number) By a triangular fuzzy number $\eta$, we mean the fuzzy variable fully determined by the triplet $A=\left(a_{1}, a_{2}, a_{3}\right)$ of crisp numbers with $a_{1}<a_{2}<a_{3}$ whose membership function is given by

$$
\mu_{A}(x)=\left\{\begin{array}{c}
\frac{x-a_{1}}{a_{2}-a_{1}} \text { if } a_{1} \leq x<a_{2} \\
\frac{x-a_{3}}{a_{2}-a_{3}} \text { if } a_{2} \leq x<a_{3} \\
0 \text { otherwise. }
\end{array}\right.
$$

Definition 3. (Trapezoidal fuzzy number) A $\operatorname{TrFN} \tilde{\xi}$ is a fuzzy number fully determined by quadruplet $\tilde{a}=\left(a_{1}, a_{2}, a_{3}, a_{4}\right)$ of crisp numbers with $r_{1} \leq r_{2} \leq r_{3} \leq r_{4}$, whose membership function is given by

$$
\mu_{\tilde{a}}(x)=\left\{\begin{array}{cc}
\frac{x-a_{1}}{a_{2}-a_{1}}, & \text { if } a_{1} \leq x \leq a_{2} ; \\
1, & \text { if } a_{2} \leq x \leq a_{3} \\
\frac{a_{4}-x}{a_{4}-a_{3}}, & \text { if } a_{3} \leq x \leq a_{4} \\
0, & \text { otherwise. }
\end{array}\right.
$$

Example. A man is driving along a highway where the speed limit is $55 \mathrm{mph}$. He tries to hold his speed at exactly $55 \mathrm{mph}$, but his car lacks 'cruise control', so the speed varies from moment to moment. If a graph of his instantaneous speed over a period of several minutes is to be plotted and the result will be in rectangular coordinates, he will get a function that looks like the curves shown in Figures 1 and 2 which represent a triangular fuzzy number and trapezoidal fuzzy number, 


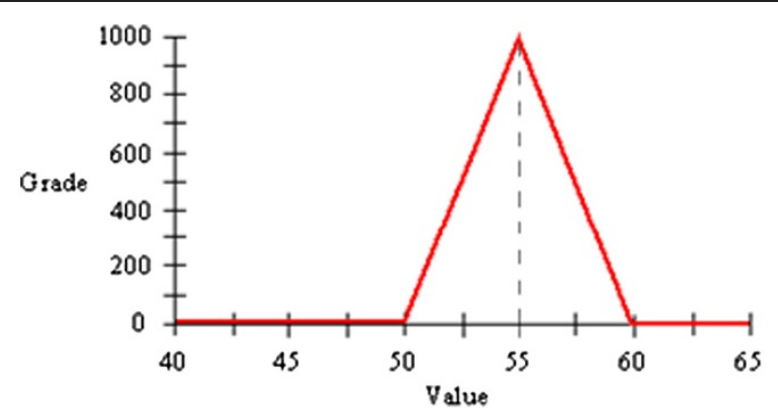

Figure 1 Triangular fuzzy number.

respectively. Crisp number $b$ can be expressed as fuzzy number $\tilde{b}$, whose membership function $\mu_{\tilde{b}}$ (Figure 3) is given by

$$
\mu_{\tilde{b}}(x)=\left\{\begin{array}{c}
1 \text { for } x=b, \\
0 \text { for } x \neq b .
\end{array}\right.
$$

Definition 4. (Credibility measure) Credibility measure was presented by Liu and Liu [17]. For a fuzzy variable $\xi$ with membership function $\mu_{\xi}(x)$ and for any set B of real numbers, credibility measure of fuzzy event $\{\xi \in B\}$ is defined as

$$
\operatorname{Cr}\{\xi \in B\}=\frac{1}{2}(\operatorname{Pos}\{\xi \in B\}+\operatorname{Nec}\{\xi \in B\}),
$$

where possibility and necessity of $\{\xi \in B\}$ are respectively defined as and

$$
\operatorname{Pos}\{\xi \in B\}=\sup _{x \in B} \mu_{\xi}(x)
$$

and

$$
\operatorname{Nec}\{\xi \in B\}=1-\sup _{x \in B^{c}} \mu_{\xi}(x) .
$$

Definition 5. [11,25]. Let $\xi$ be a fuzzy variable. Then, the expected value of $\xi$ is defined as

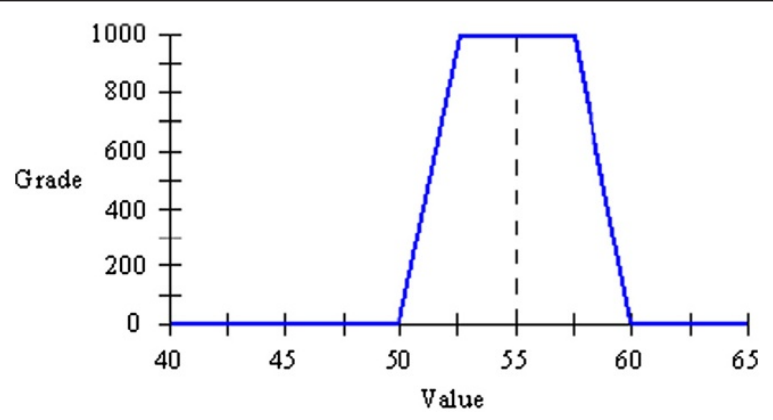

Figure 2 Trapezoidal fuzzy number. 


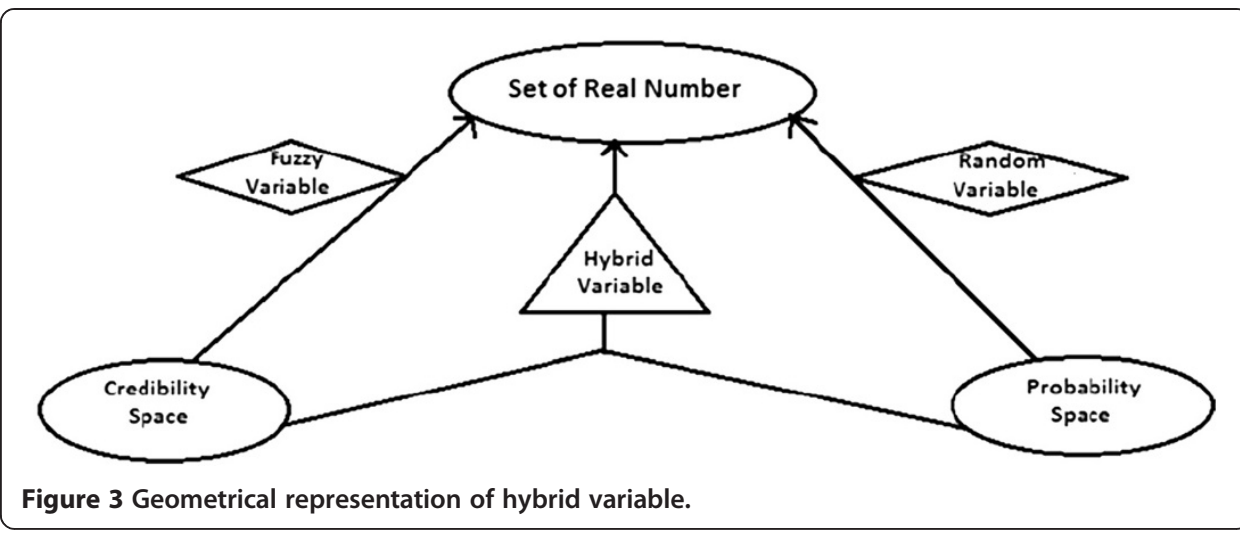

$$
E(\xi)=\int_{0}^{\infty} \operatorname{cr}\{\xi \geq r\} \mathrm{dr}-\int_{-\infty}^{0} \operatorname{cr}\{\xi \leq r\} \mathrm{dr}
$$

provided that at least one of the two integral is finite.

For example, the expected value of a triangular fuzzy variable $\left(r_{1}, r_{2}, r_{3}\right)$ is $[\xi]=\frac{r_{1}+2 r_{2}+r_{3}}{4}$.

Definition 6. [11]. Let $\xi$ be a fuzzy variable and $\alpha \in[0,1]$. Then $\xi_{\text {sup }}(\alpha)=\sup \{r: \operatorname{cr}\{\xi \geq r\} \geq \alpha\}$ is called $\alpha$-optimistic value to $\xi$, and $\xi_{\text {inf }}(\alpha)=\inf \{r: \operatorname{cr}\{\xi \leq r\} \geq \alpha\}$ is called $\alpha$-pessimistic value to $\xi$.

Example 1. Let $\xi=\left(r_{1}, r_{2}, r_{3}, r_{4}\right)$ be a trapezoidal fuzzy variable. Then, its $\alpha$-optimistic and $\alpha$-pessimistic values are

$$
\begin{aligned}
& \xi_{\text {sup }}(\alpha)=\left\{\begin{array}{c}
2 \alpha r_{3}+(1-2 \alpha) r_{4}, \text { if } \alpha \leq 0.5 \\
(2 \alpha-1) r_{1}+2(1-\alpha) r_{2}, \text { if } \alpha>0.5
\end{array}\right. \\
& \xi_{\text {inf }}(\alpha)=\left\{\begin{array}{c}
2 \alpha r_{2}+(1-2 \alpha) r_{1}, \text { if } \alpha \leq 0.5 \\
(2 \alpha-1) r_{4}+2(1-\alpha) r_{3}, \text { if } \alpha>0.5
\end{array}\right.
\end{aligned}
$$

\section{Nearest interval approximation}

The nearest interval approximation of a fuzzy number [24] $\tilde{A}$ with distance metric $d$ is given by $C_{d}(\tilde{A})=\left[C_{\mathrm{L}}-C_{\mathrm{U}}\right]$, where $C_{\mathrm{L}}=\int_{0}^{1} A_{\mathrm{L}}(\alpha) d \alpha$ and $C_{\mathrm{U}}=\int_{0}^{1} A_{\mathrm{U}}(\alpha) d \alpha$, where distance metric $d$ measure distance of $\tilde{A}$ from $C_{d}(\tilde{A})$ is given by

$$
d\left(d, C_{d}(\tilde{A})\right)=\sqrt{\int_{0}^{1}\left\{A_{\mathrm{L}}(\alpha)-C_{\mathrm{L}}\right\}^{2} d \alpha+\int_{0}^{1}\left\{A_{\mathrm{U}}(\alpha)-C_{\mathrm{U}}\right\}^{2} d \alpha}
$$

and $\left[A_{\mathrm{L}}(\alpha), A_{\mathrm{U}}(\alpha)\right.$ is the $\alpha$-cut $(0 \leq \alpha \leq 1)$ of $\tilde{A}$. For example, $\alpha$-cut of a trapezoidal fuzzy number $\left(r_{1}, r_{2}, r_{3}, r_{4}\right)$ is $\left[r_{1}+\alpha\left(r_{2}-r_{1}\right), r_{4}-\alpha\left(r_{4}-r_{3}\right)\right]$ and its interval approximation is $\left[\frac{r_{1}+r_{2}}{2}, \frac{r_{3}+r_{4}}{2}\right]$.

Theorem 1. [18] Suppose that $\xi$ is a fuzzy number with continuous membership function $\mu_{\xi}(x)$, and $r_{0}=\sup \left\{r: \mu_{\xi}(x)=1\right\}, g(x, \xi)=h(x)-\xi$. Then, we have $\operatorname{Cr}\{g(x, \xi) \geq 0\} \geq \alpha$ if and only if $h(x) \geq F_{\alpha}$, where 


$$
F_{\alpha}= \begin{cases}\inf \left\{F \mid F=\mu_{\xi}^{-1}(2 \alpha)\right\}, & \text { if } \alpha \leq 0.5, \\ \inf \left\{F \mid F=\mu_{\xi}^{-1}(2(1-\alpha)), F>r_{0}\right\} & \text { if } \alpha>0.5 .\end{cases}
$$

Theorem 2. [18] Suppose that $\xi$ is a fuzzy number with continuous membership. Function $\mu_{\xi}(x)$ and $r_{0}=\inf \left\{r: \mu_{\xi}(r)=1\right\}, g(x, \xi)=h(x)-\xi$. Then, we have $\operatorname{Cr}\{g(x, \xi) \leq 0\} \geq \alpha$ if and only if $h(x) \leq F_{\alpha}$, where

$$
F_{\alpha}= \begin{cases}\sup \left\{F \mid F=\mu_{\xi}^{-1}(2 \alpha)\right\}, & \text { if } \alpha \leq 0.5, \\ \sup \left\{F \mid F=\mu_{\xi}^{-1}(2(1-\alpha)), F<r_{0}\right\} & \text { if } \alpha>0.5 .\end{cases}
$$

Theorem 3. $[11,25]$ Assume that the function $g(x, \xi)$ can be written as

$$
g(x, \xi)=h_{1}(x) \xi_{1}+h_{2}(x) \xi_{2}+\cdots \cdots \cdots \cdots+h_{t}(x) \xi_{t}+h_{0}(x)
$$

where $\xi_{k}$ are trapezoidal fuzzy variables $\left(r_{k 1}, r_{k 2}, r_{k 3}, r_{k 4}\right), k=1,2, \ldots t$, respectively. We define two functions $h_{k}^{+}(x)=h_{k}(x) \vee 0$ and $h_{k}^{-}(x)=-\left(h_{k}(x) \vee 0\right)$ fork $=1,2, \ldots . t$. Then, we have the following:

1. When $\alpha \leq \frac{1}{2}, \operatorname{Cr}\{g(x, \xi) \leq 0\} \geq \alpha$ if and only if

$$
(1-2 \alpha) \sum_{k=1}^{t}\left[r_{k 1} h_{k}^{+}(x)-r_{k 4} h_{k}^{-}(x)\right]+2 \alpha \sum_{k=1}^{t}\left[r_{k 2} h_{k}^{+}(x)-r_{k 3} h_{k}^{-}(x)\right]+h_{0}(x) \leq 0
$$

2. When $\alpha>\frac{1}{2}, \operatorname{Cr}\{g(x, \xi) \leq 0\} \geq \alpha$ if and only if

$$
(2-2 \alpha) \sum_{k=1}^{t}\left[r_{k 3} h_{k}^{+}(x)-r_{k 2} h_{k}^{-}(x)\right]+(2 \alpha-1) \sum_{k=1}^{t}\left[r_{k 4} h_{k}^{+}(x)-r_{k 1} h_{k}^{-}(x)\right]+h_{0}(x) \leq 0 ;
$$

Corollary 1. If $\tilde{\xi}=\left(r_{1}, r_{2}, r_{3}, r_{4}\right)$ is a trapezoidal fuzzy variable and $h(x)$ is a function of $x$, then $\operatorname{Cr}\{h(x) \leq \tilde{\xi}\} \geq \alpha$ if and only if $h(x) \leq F_{\xi}$, where

$$
F_{\xi}=\left\{\begin{array}{l}
(1-2 \alpha) r_{4}+2 \alpha r_{3}, \quad \text { if } \alpha \leq \frac{1}{2} \\
2(1-\alpha) r_{2}+(2 \alpha-1) r_{1}, \quad \text { if } \alpha>\frac{1}{2}
\end{array}\right.
$$

Proof. $\operatorname{Cr}\{h(x) \leq \tilde{\xi}\} \geq \alpha \leftrightarrow \operatorname{Cr}\left\{\tilde{\xi}^{\prime}+h(x) \leq 0\right\} \geq \alpha$, where $\tilde{\xi}^{\prime}=-\tilde{\xi}=\left(-r_{4},-r_{3},-r_{2},-r_{1}\right)$.

Then, from the above theorem, it follows that this inequality holds if and only if

1. $(1-2 \alpha)\left(-r_{4}\right)+2 \alpha\left(-r_{3}\right)+h(x) \leq 0$, if $\alpha \leq \frac{1}{2}$;

2. $(2-2 \alpha)\left(-r_{2}\right)+(2 \alpha-1)\left(-r_{1}\right)+h(x) \leq 0$, if $\alpha>\frac{1}{2}$.

Hence, the corollary follows.

Corollary 2. If $\tilde{\xi}=\left(r_{1}, r_{2}, r_{3}, r_{4}\right)$ is a trapezoidal fuzzy variable and $h(x)$ is a function of $x$, then $\operatorname{Cr}\{h(x) \geq \tilde{\xi}\} \geq \alpha$ if and only if $h(x) \geq F_{\xi}$, where 


$$
F_{\xi}=\left\{\begin{array}{c}
(1-2 \alpha) r_{1}+2 \alpha r_{2}, \text { if } \alpha \leq \frac{1}{2} ; \\
(2-2 \alpha) r_{3}+(2 \alpha-1) r_{4}, \quad \text { if } \alpha>\frac{1}{2} .
\end{array}\right.
$$

Proof. $\operatorname{Cr}\{h(x) \geq \tilde{\xi}\} \geq \alpha \Leftrightarrow \operatorname{Cr}\left\{\tilde{\xi}^{\prime}-h(x) \leq 0\right\} \geq \alpha$, where $\tilde{\xi}^{\prime}=-\tilde{\xi}=\left(-r_{4},-r_{3},-r_{2},-r_{1}\right)$.

Then, from the above theorem, it follows that this inequality holds if and only if

1. $(1-2 \alpha) r_{1}+2 \alpha r_{2}-h(x) \leq 0$, if $\alpha \leq \frac{1}{2}$;

2. $(2-2 \alpha) r_{3}+(2 \alpha-1) r_{4}-h(x) \leq 0$, if $\alpha>\frac{1}{2}$.

Hence, the corollary follows.

Corollary 3. If $\tilde{\xi}=\left(r_{1}, r_{2}, r_{3}, r_{4}\right)$ and $\tilde{\eta}=\left(t_{1}, t_{2}, t_{3}, t_{4}\right)$ are trapezoidal fuzzy variable and $h(x) \geq 0 \forall x$, then $\operatorname{Cr}\{h(x) \tilde{\xi} \leq \tilde{\eta}\} \geq \alpha$ if and only if

1. $(1-2 \alpha) h(x) r_{1}+2 \alpha h(x) r_{2}-h(x) \leq(1-2 \alpha) t_{4}+2 \alpha t_{3}$, if $\alpha \leq \frac{1}{2}$;

2. $(2-2 \alpha) h(x) r_{3}+(2 \alpha-1) h(x) r_{4}-h(x) \leq(2-2 \alpha) t_{2}+(2 \alpha-1) t_{1}$, if $\alpha>\frac{1}{2}$.

Proof. $\operatorname{Cr}\{h(x) \tilde{\xi} \leq \tilde{\eta}\} \geq \alpha \Leftrightarrow \operatorname{Cr}\{h(x) \tilde{\xi}-\tilde{\eta} \leq 0\} \geq \alpha \Leftrightarrow \operatorname{Cr}\left\{h(x) \tilde{\xi}+\tilde{\eta}^{\prime} \leq 0\right\} \geq \alpha$, where $\tilde{\eta}^{\prime}=-$ $\tilde{\eta}=\left(-t_{4},-t_{3},-t_{2},-t_{1}\right)$. Then, from the above theorem, it follows that this inequality holds if and only if

1. $(1-2 \alpha)\left(h(x) r_{1}-t_{4}\right)+2 \alpha\left(h(x) r_{2}-t_{3}\right) \leq 0$, if $\alpha \leq \frac{1}{2}$;

2. $(2-2 \alpha)\left(h(x) r_{3}-t_{2}\right)+(2 \alpha-1)\left(h(x) r_{4}-t_{1}\right)+h(x) \leq 0$, if $\alpha>\frac{1}{2}$

Hence, the corollary follows.

It is obvious that these three corollaries help us to determine crisp equivalences of various inequalities with fuzzy parameters.

\section{Random variable}

For the probability spaces $(\Omega, S, P)$ where $\Omega$ is a set of elementary events, $S$ is a set of all events ( $a \sigma$-field of events) and $P: S \rightarrow[0,1]$ is a probability function, the mapping $\widehat{X}:(\Omega, S, P) \rightarrow \mathfrak{N}$ is called a random variable.

By the probability distribution function of the random variable $\widehat{X}$, we mean a function $F(x)=P\{\omega: \hat{X}(\omega) \leq X\}$ for all $x \in N$ with $F(-\infty)=0$ and $F(\infty)=1$.

\section{Hybrid variable}

Recall that a random variable is a measurable function from a probability space to the set of real number and that a fuzzy variable is a function from a credibility space to the set of real numbers. In order to describe a quantity with both fuzziness and randomness, we introduce the concept of hybrid variable as follows:

Definition 7. [11] A hybrid variable is a measurable function from a chance space $(\Theta, P, \mathrm{Cr}) \times(\Omega, S, \operatorname{Pr})$ to real numbers, i.e., for any Borel set B of real numbers, the set 


$$
\{\xi \in B\}=\{(\theta, \omega) \in \Theta \times \Omega: \xi(\theta, \omega) \in B\}
$$

is an event.

Remark 1. [11] A hybrid variable degenerates to a fuzzy variable if the value of $\xi(\theta, \omega)$ does not vary with $\omega$. For example, $\xi(\theta, \omega)=\theta, \xi(\theta, \omega)=\theta^{2}+1, \xi(\theta, \omega)=\sin \theta$.

Remark 2. [11] A hybrid variable degenerate to a random variable if the value of $\xi(\theta, \omega)$ does not vary with $\theta$. For example, $\xi(\theta, \omega)=\omega, \xi(\theta, \omega)=\omega^{2}+1, \xi(\theta, \omega)=\sin \omega$.

Remark 3. A hybrid variable $\xi(\theta, \omega)$ may also be regarded as a function from a credibility space $(\Theta, \mathcal{P}, \mathrm{Cr})$ to the set $\{\xi(\theta,) \mid. \theta \in \Theta\}$ of random variable. Thus, $\xi$ is a random fuzzy variable defined by Liu [11].

Remark 4. A hybrid variable $\xi(\theta, \omega)$ may also be regarded as a function from a probability space $(\Omega, \mathcal{A}, \operatorname{Pr})$ to the set $\{\xi($., $\omega) \mid \omega \in \Omega\}$ of fuzzy variables. If $\operatorname{Cr}\{\xi(., \omega) \in B\}$ is a measurable function of $\omega$ for any Borel set B of real number, then $\xi$ is a fuzzy random in the sense of [17].

Definition 8. [17] Then, a chance measure of an event $\wedge$ is defined as

$$
\operatorname{ch}(\wedge)=\left\{\begin{array}{cl}
\sup _{\theta \in \Theta}(\operatorname{Cr}\{\theta\} \wedge \operatorname{Pr}\{\wedge(\theta)\}), & \text { if } \sup _{\theta \in \Theta}(\operatorname{Cr}\{\theta\} \wedge \operatorname{Cr}\{\wedge(\theta)\})<0.5 \\
1-\sup _{\theta \in \Theta}(\operatorname{Cr}\{\theta\} \wedge \operatorname{Pr}\{\wedge(\theta)\}) & \text { if } \sup _{\theta \in \Theta}(\operatorname{Cr}\{\theta\} \wedge \operatorname{Pr}\{\wedge(\theta)\}) \geq 0.5 .
\end{array}\right.
$$

Then $\operatorname{ch}(\phi)=0, \operatorname{ch}\{\Theta \times \Omega\}=1,0 \leq \operatorname{ch}\{\wedge\} \leq 1$ for any event $\wedge$.

Example 2. If $\tilde{\mathbf{a}}$ is a fuzzy variable and $\hat{\eta}$ is a random variable, then the sum $\tilde{\hat{\xi}}=\tilde{\mathbf{a}}+\hat{\eta}$ is a hybrid variable, i.e., if $f: \mathfrak{N}^{2} \rightarrow \mathfrak{N}$ is a measurable function, then $\tilde{\hat{\xi}}=f(\tilde{\mathbf{a}}, \hat{\eta})$ is a hybrid variable. Now, suppose that $\tilde{\mathbf{a}}$ has a membership function $\mu$, and $\hat{\eta}$ has a probability density function $\phi$. Then, for any Borel set B of real numbers, we have

$$
\operatorname{ch}\{f(\tilde{a}, \hat{\eta}) \in B\}= \begin{cases}\sup _{x}\left[\frac{\mu(x)}{2} \wedge \int_{f(x, y) \in B} \phi(\mathrm{y}) \mathrm{dy}\right], & \text { if } \sup _{x}\left[\frac{\mu(x)}{2} \wedge \int_{f(x, y) \in B} \phi(y) d y\right]<0.5 \\ 1-\sup _{x} \sup _{x}\left[\frac{\mu(x)}{2} \wedge \int_{f(x, y) \in B} \phi(y) d y\right], & \text { if } \sup _{x}\left[\frac{\mu(x)}{2} \wedge \int_{f(x, y) \in B} \phi(y) d y\right] \geq 0.5 .\end{cases}
$$

Definition 9. [17] Let $\tilde{\hat{\xi}}$ be a hybrid variable. Then, the expected value of $\tilde{\hat{\xi}}$ is defined by

$$
E[\tilde{\hat{\xi}}]=\int_{0}^{+\infty} \operatorname{ch}\{\tilde{\hat{\xi}} \geq r\} d r-\int_{-\infty}^{0} \operatorname{ch}\{\tilde{\hat{\xi}} \geq r\} d r
$$

provided that at least one of the integrals is finite.

Example 3. For the hybrid variable $\tilde{\hat{\xi}}=\tilde{\mathbf{a}}+\hat{\eta}$, expected value of $\tilde{\hat{\xi}}$ is $E(\tilde{\hat{\xi}})=E(\tilde{\mathbf{a}})+\mathbf{E}$ $(\hat{\eta})$. For example, if $\tilde{\mathbf{a}}=\left(t_{1}, t_{2}, t_{3}\right)$ is the triangular fuzzy number and $\hat{\eta}=\mathcal{N}\left(\mu, \sigma^{2}\right)$ is normally distributed as random variable. Then, $E(\tilde{\hat{\xi}})=\frac{t_{1}+2 t_{2}+t_{3}}{4}+\mu$. 


\section{Description of the problem}

STP is a problem of transporting goods from some sources to some destinations through some conveyances (modes of transportation), and the main objective is to find the optimal transportation plan so that the total transportation cost is minimum. Also, the goods transported through each source cannot exceed its supply capacity, the requirements of each destination must be satisfied, and the total transported amount must not exceed the capacity of conveyances. In this manuscript, we impose a desired total safety factor for the whole transportation system. The decision of future transportation planning is generally founded upon the past record. However, the available data from previous experiments are not always precise; those are often imprecise due to uncertainty in judgment, fluctuating financial market, linguistic information, imperfect statistical analysis, insufficient information, etc. For example, transportation cost depends on the fuel price, labor charges, tax charges, etc., each of which are fluctuating from time to time. Similarly, the supply of a source cannot be always exact because it depends upon the availability of manpower, raw materials, market competition, product demands, etc. Fuzzy set theory and random set theory are most widely used and are successfully applied tools to deal with uncertainty. In the next section, we formulate five STPs with safety constraints and different uncertain (fuzzy, random, hybrid) parameters.

\section{Model formulation}

Sometimes, information about supplies at plants, demands at destination, and conveyance capacity are not known precisely, i.e., these amounts are erstwhile vague in nature. For this reason, we consider here that the supplies at plants, demands at destination, and conveyance capacity are all fuzzy in nature.

Model 1: solid transportation problem with hybrid penalties, fuzzy resources, demands, conveyance capacities, and without safety factor

To formulate the model, we assume that there are no risks to transport the commodities from plants to destination by different conveyances, i.e., all routes are totally safe for the transport of goods, and unit transportation cost is a hybrid variable.

$$
\operatorname{Min}(Z)=\sum_{i=1}^{m} \sum_{j=1}^{n} \sum_{k=1}^{K} \tilde{\hat{C}}_{i j k} x_{i j k}
$$

subject to constraints

$$
\begin{aligned}
& \sum_{j=1}^{n} \sum_{k=1}^{k} x_{i j k} \leq \tilde{a}_{i}, \quad i=1,2, \ldots, m, \\
& \sum_{i=1}^{m} \sum_{k=1}^{k} x_{i j k} \geq \tilde{b}_{j}, \quad j=1,2, \ldots, n, \\
& \sum_{j=1}^{n} \sum_{k=1}^{k} x_{i j k} \leq \tilde{e}_{k}, \quad k=1,2, \ldots, K, \\
& x_{i j k} \geq 0, \quad \forall i, j, k,
\end{aligned}
$$

where $\tilde{a}_{i}$ denotes the fuzzy amount of the product available at the $i$ th origin, $\tilde{b}_{j}$ denotes the fuzzy demand of the product of the $j$ th destination, $\tilde{e}_{k}$ denotes the fuzzy transportation capacity of conveyance $k, \tilde{\hat{C}}_{i j k}$ denotes the hybrid unit transportation penalty from the $i$ th origin to the $j$ th destination via the $k$ th conveyance for the objective function. 
The penalty could represent transportation cost or quantity of goods delivered or underused capacity, etc. Here, the penalties represent per unit transportation costs.

\section{Solution methodology (expected value model)}

Liu and Liu [17] presented a spectrum of expected value model of fuzzy programming to obtain the optimum expected value of objective function under some expected constraints. Considering $\tilde{\hat{C}}_{i j k}$ as $\tilde{\hat{C}}_{i j k}=C_{i j k}+\hat{C}_{i j k}$ and constructing the expected value model $[17,18,26]$ for the model I, we have the corresponding crisp form as

$$
\operatorname{Min} E(Z)=\sum_{i=1}^{m} \sum_{j=1}^{n} \sum_{k=1}^{K} E\left(\tilde{\hat{C}}_{i j k}\right) x_{i j k}
$$

subject to constraints

$$
\begin{aligned}
& \sum_{j=1}^{n} \sum_{k=1}^{k} x_{i j k} \leq E\left(\tilde{a}_{i}\right), \quad i=1,2, \ldots, m, \\
& \sum_{i=1}^{m} \sum_{k=1}^{k} x_{i j k} \geq E\left(\tilde{b}_{j}\right), \quad j=1,2, \ldots, n, \\
& \sum_{j=1}^{n} \sum_{k=1}^{k} x_{i j k} \leq E\left(\tilde{e}_{k}\right), \quad k=1,2, \ldots, K, \\
& x_{i j k} \geq 0, \quad \forall i, j, k .
\end{aligned}
$$

Model 2: solid transportation problem with crisp penalties, fuzzy resources, demands, conveyance capacities, and desired safety measure as crisp

To formulate the model, we assume that all transportation routes are not equally safe to transport the commodity from the source to the destination by different conveyances, and we consider a crisp safety factor for each transportation:

$$
\operatorname{Min}(Z)=\sum_{i=1}^{m} \sum_{j=1}^{n} \sum_{k=1}^{K} C_{i j k} x_{i j k}
$$

subject to the constraint

$$
\sum_{i=1}^{m} \sum_{j=1}^{n} \sum_{k=1}^{K} S_{i j k} y_{i j k} \geq B
$$

with constraints (2), (3), and (4),

$$
x_{i j k} \geq 0, \quad \forall i, j, k
$$

where $S_{i j k}$ is the safety factor when an item is transformed from the $i$ th origin to the $j$ th destination by the $k$ th conveyance. If an item is transported from source $i$ to destination $j$ by conveyance $k$, then the safety factor $S_{i j k}$ is considered. This implies that if $x_{i j k}>0$, then we consider the safety factor for this route as a part of the safety constraint. Thus, for the convenience of modeling, the following notation is introduced:

$$
y_{i j k}= \begin{cases}1 & \text { for } x_{i j k}>0 \\ 0 & \text { otherwise }\end{cases}
$$

and $B$ is the desired safety measure for the whole transportation system. 


\section{Solution methodology}

$$
\operatorname{Min}(Z)=\sum_{i=1}^{m} \sum_{j=1}^{n} \sum_{k=1}^{K} C_{i j k} x_{i j k}
$$

subject to constraints

$$
\begin{aligned}
& \operatorname{Cr}\left(\sum_{j=1}^{n} \sum_{k=1}^{k} x_{i j k} \leq \tilde{a}_{i}\right) \geq \alpha_{i}, \quad i=1,2, \ldots, m, \\
& \operatorname{Cr}\left(\sum_{i=1}^{m} \sum_{k=1}^{k} x_{i j k} \geq \tilde{b}_{j}\right) \geq \beta_{j}, \quad j=1,2, \ldots, n, \\
& \operatorname{Cr}\left(\sum_{j=1}^{n} \sum_{k=1}^{k} x_{i j k} \leq \tilde{e}_{k}\right) \geq \gamma_{k}, \quad i=1,2, \ldots, k,
\end{aligned}
$$

and (9)

$$
x_{i j k} \geq 0, \quad \forall i, j, k .
$$

\section{Crisp equivalences}

$$
\operatorname{Min}(Z)=\sum_{i=1}^{m} \sum_{j=1}^{n} \sum_{k=1}^{K} C_{i j k} x_{i j k}
$$

subject to constraints

$$
\begin{aligned}
& \sum_{j=1}^{n} \sum_{k=1}^{k} x_{i j k} \leq F_{\alpha_{i}}, \quad i=1,2, \ldots, m, \\
& \sum_{i=1}^{m} \sum_{k=1}^{k} x_{i j k} \geq F_{\beta_{j}}, \quad j=1,2, \ldots, n, \\
& \sum_{j=1}^{n} \sum_{k=1}^{k} x_{i j k} \leq F_{\gamma_{k}}, \quad k=1,2, \ldots, K,
\end{aligned}
$$

and (9)

$$
x_{i j k} \geq 0, \quad \forall i, j, k \text {. }
$$

where $\tilde{a}_{i}=\left(a_{i}^{1}, a_{i}^{2}, a_{i}^{3}, a_{i}^{4}\right), \tilde{b}_{j}=\left(b_{j}^{1}, b_{j}^{2}, b_{j}^{3}, b_{j}^{4}\right), \tilde{e}_{k}=\left(e_{k}^{1}, e_{k}^{2}, e_{k}^{3}, e_{k}^{4}\right)$ and

$$
\begin{aligned}
& F_{\alpha_{i}}=a_{i_{\text {sup }}}\left(\alpha_{i}\right)=\left\{\begin{array}{l}
\left(1-2 \alpha_{i}\right) a_{i}^{4}+2 \alpha_{i} a_{i}^{3}, \quad \text { if } \alpha_{i} \leq 0.5 ; \\
2\left(1-\alpha_{i}\right) a_{i}^{2}+\left(2 \alpha_{i}-1\right) a_{i}^{1}, \quad \alpha_{i}>0.5 .
\end{array}\right. \\
& F_{\beta_{j}}=a_{j_{\text {inf }}}\left(\beta_{j}\right)=\left\{\begin{array}{l}
\left(1-2 \beta_{j}\right) b_{j}^{1}+2 \beta_{j} b_{j}^{2}, \quad \text { if } \alpha_{i} \leq 0.5 ; \\
2\left(1-\beta_{j}\right) b_{j}^{3}+\left(2 \beta_{j}-1\right) b_{j}^{4}, \alpha_{i}>0.5 .
\end{array}\right. \\
& F_{\gamma_{k}}=a_{k_{\text {sup }}}\left(\alpha_{i}\right)=\left\{\begin{array}{lc}
\left(1-2 \gamma_{k}\right) e_{k}^{4}+2 \gamma_{k} e_{i}^{3}, & \text { if } \alpha_{i} \leq 0.5 ; \\
2\left(1-\gamma_{k}\right) e_{k}^{2}+\left(2 \gamma_{k}-1\right) e_{i}^{1}, & \alpha_{i}>0.5 .
\end{array}\right.
\end{aligned}
$$

Model 3: solid transportation problem with random penalties, fuzzy resources, demands, conveyance capacities, and DSM as random

Here, we formulate the respective model by taking unit transportation cost, desired total safety factor, and safety factor as a random variables because it may happen that the safety factor is uncertain, not precisely known, but some past data about it is available:

$$
\operatorname{Min}(Z)=\sum_{i=1}^{m} \sum_{j=1}^{n} \sum_{k=1}^{K} \hat{C}_{i j k} x_{i j k}
$$


subject to the constraint

$$
\sum_{i=1}^{m} \sum_{j=1}^{n} \sum_{k=1}^{K} \hat{S}_{i j k} y_{i j k} \geq \hat{B}
$$

with constraints (2), (3), and (4),

$$
x_{i j k} \geq 0, \quad \forall i, j, k,
$$

where $\hat{C}_{i j k}$ are random unit transportation cost from the $i$ th origin to the $j$ th destination by the $k$ th conveyance, $\hat{S}_{i j k}$ is the random safety factor for a particular route, and $\hat{B}$ is the desired total random safety factor for the whole transportation system.

\section{Solution methodology (an approach using interval approximation of fuzzy number)}

Let us denote the interval approximations of $\tilde{a}_{i}, \tilde{b}_{j}, \tilde{e}_{k}$, and $\tilde{B}$ obtained using $\alpha$-cut of these fuzzy numbers are $C_{d}\left(\tilde{a}_{i}\right)=\left[a_{i L}, a_{i U}\right], C_{d}\left(\tilde{b}_{j}\right)=\left[b_{j L}, b_{j u}\right], C_{d}\left(e_{k}\right)=\left[e_{k L}, e_{k U}\right]$, respectively. Then, using these interval approximations and predetermined probability confidence level $\delta$ for the constraint (11), the above model becomes

$$
\operatorname{Min}(Z)=\sum_{i=1}^{m} \sum_{j=1}^{n} \sum_{k=1}^{K} \hat{C}_{i j k} x_{i j k}
$$

subject to constraints

$$
\begin{aligned}
& \sum_{j=1}^{n} \sum_{k=1}^{k} x_{i j k} \leq C_{d}\left(\tilde{a}_{i}\right) \\
& \sum_{i=1}^{m} \sum_{k=1}^{k} x_{i j k} \geq C_{d}\left(\tilde{b}_{j}\right) \\
& \sum_{j=1}^{n} \sum_{k=1}^{k} x_{i j k} \leq C_{d}\left(\tilde{e}_{k}\right) \\
& \operatorname{Prob}\left[\sum_{i=1}^{m} \sum_{j=1}^{n} \sum_{k=1}^{K} \hat{S}_{i j k} y_{i j k} \geq \hat{B}\right] \geq \epsilon,
\end{aligned}
$$

$x_{i j k} \geq 0, \forall i, j, k$.

\section{Crisp equivalences}

We assumed that all $\hat{C}_{i j k}$ are mutually independent normally distributed random variables with known mean $E\left(\hat{C}_{i j k}\right)=\bar{C}_{i j k}$ and variance $\operatorname{var}\left(\hat{C}_{i j k}\right)$. Then, $Z$ will also be a normally distributed random variable with mean $\bar{z}=\sum_{i=1}^{m} \sum_{j=1}^{n} \sum_{k=1}^{K} \bar{C}_{i j k} x_{i j k}$ and var $(z)=X^{T} V X$, where $V$ is the covariance matrix of $\hat{C}_{i j k}$, where

$$
V_{i j}=\left(\begin{array}{cllc}
\operatorname{var}\left(\hat{C}_{i j 1}\right) & \operatorname{cov}\left(\hat{C}_{i j 1}, \hat{C}_{i j 2}\right) & \ldots & \operatorname{cov}\left(\hat{C}_{i j 1}, \hat{C}_{i j k}\right) \\
\operatorname{cov}\left(\hat{C}_{i j 2}, \hat{C}_{i j 1}\right) & \operatorname{var}\left(\hat{C}_{i j 2}\right) & \ldots & \operatorname{cov}\left(\hat{C}_{i j 2}, \hat{C}_{i j k}\right) \\
\ldots & \ldots & \ldots & \ldots \\
\operatorname{cov}\left(\hat{C}_{i j k}, \hat{C}_{i j 1}\right) & \operatorname{cov}\left(\hat{C}_{i j k}, \hat{C}_{i j 2}\right) & \ldots & \operatorname{var}\left(\hat{C}_{i j k}\right)
\end{array}\right)
$$

For $i=1,2, \ldots, m ; j=1,2, \ldots, n$; and $k=1,2, \ldots, K$.

Then, a new deterministic nonlinear objective function for minimization can be formulated as

$$
z(X)=\lambda_{1} \bar{z}+\lambda_{2} \sqrt{X^{T} V X}
$$


where $\lambda_{1}$ and $\lambda_{2}$ are nonnegative constants whose values indicate the relative importance of mean and standard deviation of $z$ for minimization. Thus, $\lambda_{2}=0$ (i. e., $\lambda_{1}=1$ ) indicates that only the expected value (mean value) of the objective function is to be minimized without caring for the standard deviation (SD) of the objective function. Similarly, $\lambda_{1}=0 \lambda$ indicates that only the SD, i.e., the variability of the objective function about its mean is to be minimized without caring of for its mean. $\lambda_{1}=\lambda_{2}=1$ indicates that equal importance is given to the minimization of both the mean and SD of the objective function.

If the r.v. $\hat{C}_{i j k}$ are independent, then $Z(X)$ reduces to

$$
Z(X)=\lambda_{1} \sum_{i=1}^{m} \sum_{j=1}^{n} \sum_{k=1}^{K} \bar{C}_{i j k} x_{i j k}+\lambda_{2} \sqrt{\sum_{i=1}^{m} \sum_{j=1}^{n} \sum_{k=1}^{K} \operatorname{var}\left(\hat{C}_{i j k}\right) x_{i j k}^{2}}
$$

If $\varepsilon$ are the probabilities of nonviolation of the constraint (16) then the constraint can be written as

$$
\begin{aligned}
& \operatorname{Prob}\left[\sum_{i=1}^{m} \sum_{j=1}^{n} \sum_{k=1}^{K} \hat{S}_{i j k} y_{i j k} \geq \hat{B}\right] \geq \epsilon, \\
& \Rightarrow \operatorname{Prob}\left[\sum_{i=1}^{m} \sum_{j=1}^{n} \sum_{k=1}^{K} \hat{S}_{i j k} y_{i j k}-\hat{B} \geq 0\right] \geq \epsilon, \\
& \Rightarrow \operatorname{Prob}[\hat{Q} \geq 0] \geq \epsilon, \text { where } \hat{Q}=\sum_{i=1}^{m} \sum_{j=1}^{n} \sum_{k=1}^{K} \hat{S} i j k y_{i j k}-\hat{B} \\
& \Rightarrow \operatorname{Prob}\left[\frac{\hat{Q}-E(\hat{Q})}{\operatorname{Var}(\hat{Q})} \geq-\frac{E(\hat{Q})}{\operatorname{Var}(\hat{Q})}\right] \geq \epsilon, \\
& \Rightarrow \operatorname{Prob}[\hat{T} \geq-K] \geq \epsilon, \\
& \Rightarrow \operatorname{Prob}[\hat{T} \leq K] \geq \epsilon,
\end{aligned}
$$

where $\hat{T}=\frac{\hat{Q}-E(\hat{Q})}{\operatorname{Var}(\hat{Q})}$ are the standard normal variate and $K=\frac{E(\hat{Q})}{\operatorname{Var}(\hat{Q})}$

$$
\begin{aligned}
& \Rightarrow E(\hat{Q}) \geq \lambda \operatorname{Var}(\hat{Q}) . \\
& \Rightarrow \sum_{i=1}^{m} \sum_{j=1}^{n} \sum_{k=1}^{K} E\left(\hat{S}_{i j k}\right) y_{i j k}-E(\hat{B}) \geq \lambda\left[\sum_{i=1}^{m} \sum_{j=1}^{n} \sum_{k=1}^{K} \operatorname{Var}\left(\hat{S}_{i j k}\right) y_{i j k}-\operatorname{Var}(\hat{B})\right] .
\end{aligned}
$$

where $\lambda$ be the real number such that $\operatorname{Prob}[\hat{T} \geq \lambda]=\epsilon$.

Now, denote the left-hand side expressions of the constraints (19), (20), (21), and (23) by $S_{i}, D_{j}, E_{k}$. The right-hand side expressions of the constraints are interval numbers. Now, using the idea of possibility degree of interval numbers [27] that represent a certain degree by which one interval is larger or smaller than the other, we define the possibility degree of satisfaction of these constraints as follows:

$$
\begin{gathered}
P_{S_{i} \leq\left[a_{i L}, a_{i U}\right]}=\left\{\begin{array}{lc}
1 & S_{i} \leq a_{i L} ; \\
\frac{a_{i U}-S_{i}}{a_{i U}-a_{i L}} & a_{i L} \leq S_{i} \leq a_{i U} ; \\
0 & S_{i}>a_{i U} .
\end{array}\right. \\
P_{D_{j} \geq\left[b_{j L}, b_{j U}\right]}=\left\{\begin{array}{lc}
0 & D_{j} \leq b_{j L} ; \\
\frac{D_{j}-b_{i L}}{b_{j U}-a_{j L}} & a_{j L} \leq S_{j} \leq a_{j U} ; \\
1 & D_{j}>a_{j U} .
\end{array}\right.
\end{gathered}
$$




$$
P_{E_{k} \leq\left[e_{k L}, e_{k U}\right]}=\left\{\begin{array}{lc}
1 & E_{k} \leq e_{k L} ; \\
\frac{e_{k U}-e_{k}}{e_{k U}-e_{k L}} & e_{k L} \leq E_{k} \leq e_{k U} \\
0 & E_{i}>e_{k U} .
\end{array}\right.
$$

Now, for a predetermined possibility degree of satisfaction, $\alpha_{i}, \beta_{j}, \gamma_{k}$ and $\epsilon\left(0 \leq \alpha_{i}, \beta_{j}, \gamma_{k} \leq 1\right)$, respectively, for the constraints, i.e., $P_{S_{i} \leq\left[a_{i L}, a_{i l}\right]} \geq \alpha_{i}, P_{D_{j} \geq\left[b_{j l}, b_{j l}\right]} \geq \beta_{j}, P_{E_{k} \leq\left[e_{k L}, e_{k U}\right]} \geq \gamma_{k} \forall i, j, k$, then the equivalent deterministic inequalities of the respective constraints are obtained as follows:

$$
\begin{aligned}
& S_{i} \leq a_{i U}-\alpha_{i}\left(a_{i U}-a_{i L}\right)=a_{i c} \text { (say), } \\
& D_{j} \geq b_{j L}+\beta_{j}\left(b_{j U}-b_{j L}\right)=b_{j c}, \\
& E_{k} \leq e_{k U}-\gamma_{k}\left(e_{k U}-e_{k L}\right)=e_{k c},
\end{aligned}
$$

Now, using the deterministic form (23) of the objective function (17) and the constraints (27), the deterministic form of problems (17) to (21) becomes

$$
\operatorname{Min} z(x)=\lambda_{1} \sum_{i=1}^{m} \sum_{j=1}^{n} \sum_{k=1}^{K} \bar{C}_{i j k} x_{i j k}+\lambda_{2} \sqrt{\sum_{i=1}^{m} \sum_{j=1}^{n} \sum_{k=1}^{K} \operatorname{var}\left(\hat{C}_{i j k}\right) x_{i j k}^{2}},
$$

subject to constraints (25) and (24)

$$
x_{i j k} \geq 0, \quad \forall i, j, k \text {. }
$$

Model 4: solid transportation problem with fuzzy penalties, resources, demands, conveyance capacities, and DSM as fuzzy

In this section, we formulate the model by taking the unit transportation cost, desired total safety factor, and safety factor as fuzzy numbers because it may happen that the safety factor is vague in nature, i.e., imprecisely known:

$$
\operatorname{Min}(Z)=\sum_{i=1}^{m} \sum_{j=1}^{n} \sum_{k=1}^{K} \tilde{C}_{i j k} x_{i j k}
$$

subject to the constraint

$$
\sum_{i=1}^{m} \sum_{j=1}^{n} \sum_{k=1}^{K} \tilde{S}_{i j k} y_{i j k} \geq \tilde{B}
$$

with constraints (2), (3), and (4),

$$
x_{i j k} \geq 0, \quad \forall i, j, k,
$$

where $\tilde{C}_{i j k}$ is the fuzzy unit transportation cost from the $i$ th origin to the $j$ th destination by the $k$ th conveyance, $\tilde{S}_{i j k}$ is the fuzzy safety factor for a particular route, and $\tilde{B}$ is the desired total fuzzy safety measure for the whole transportation system.

\section{Solution methodology (chance-constrained programming)}

Chance-constrained programming technique was introduced by Charnes and Cooper [15] for stochastic programming. Chance-constrained programming in a fuzzy case was developed by Liu and Iwamura [16], Liu [25], Yang and Liu [18], and many more authors. This method is used to solve the problems with chance constraints. In this 
method, the uncertain constraints are allowed to be violated such that constraints must be satisfied at some chance (or confidence) level. Applying this idea, we reformulate the above problem as follows:

Min $\bar{z}$, subject to constraints,

$$
\begin{aligned}
& \operatorname{Cr}\left\{\sum_{i=1}^{m} \sum_{j=1}^{n} \sum_{k=1}^{K} \tilde{C}_{i j k} x_{i j k} \leq \bar{z}\right\} \geq \eta \\
& \operatorname{Cr}\left\{\sum_{i=1}^{m} \sum_{j=1}^{n} \sum_{k=1}^{K} \tilde{S}_{i j k} y_{i j k} \geq \tilde{B}\right\} \geq \delta, \\
& \operatorname{Cr}\left\{\sum_{j=1}^{n} \sum_{k=1}^{k} x_{i j k} \leq \tilde{a}_{i}\right\} \geq \alpha_{i} \\
& \operatorname{Cr}\left\{\sum_{i=1}^{m} \sum_{k=1}^{k} x_{i j k} \geq \tilde{b}_{j}\right\} \geq \beta_{j} \\
& \operatorname{Cr}\left\{\sum_{j=1}^{n} \sum_{k=1}^{k} x_{i j k} \leq \tilde{e}_{k}\right\} \geq \gamma_{k} \\
& x_{i j k} \geq 0, \quad \forall i, j, k .
\end{aligned}
$$

where $\eta$ indicates that we are going to optimize the $\eta$-critical value of the objective $z$, and $\delta$ indicates the credibility level of satisfaction of the safety constraint.

\section{Crisp equivalence}

$\tilde{C}_{i j k}=\left(C_{i j k}^{1}, C_{i j k}^{2}, C_{i j k}^{3}, C_{i j k}^{4}\right), \tilde{a}_{i}=\left(a_{i}^{1}, a_{i}^{2}, a_{i}^{3}, a_{i}^{4}\right), \tilde{b}_{j}=\left(b_{j}^{1}, b_{j}^{2}, b_{j}^{3}, b_{j}^{4}\right), \tilde{e}_{k}=\left(e_{k}^{1}, e_{k}^{2}, e_{k}^{3}, e_{k}^{4}\right)$,

$\tilde{B}=\left(B_{1}, B_{2}, B_{3}, B_{4}\right)$ are trapezoidal fuzzy numbers for all $i, j$, and $k$.

Now, since $\tilde{C}_{i j k}$ are trapezoidal fuzzy numbers and $x_{i j k}$ is 0 for all $i, j, k$, so $Z(x)=$ $\sum_{i=1}^{m} \sum_{j=1}^{n} \sum_{k=1}^{K} \tilde{C}_{i j k} x_{i j k}$ are also trapezoidal fuzzy numbers for any feasible solution $x$ and given by $Z(x)=\left(r_{1}(x), r_{2}(x), r_{3}(x), r_{4}(x)\right)$. Then, the objective in the above model (18), i.e., Min $\bar{Z}$ s.t. $\operatorname{Cr}\{Z(x) \leq \bar{Z}\} \geq \eta$ can be equivalently computed as $\bar{Z}=\inf$ $\{\mathrm{r}: \operatorname{Cr}\{Z(x) \leq \bar{Z}\} \geq \eta\}$ which is nothing but $\eta$ - pessimistic value of $Z\left(Z_{\text {inf }}(\eta)\right)$ and so is equal to $z^{\prime}(x), z$ where

$$
\begin{aligned}
Z^{\prime}(x) & =\left\{\begin{array}{cc}
(1-2 \eta) r_{1}(x)+2 \eta r_{2}(x) & \text { if } \alpha \leq 0.5, \\
2(1-\eta) r_{3}(x)+(2 \eta-1) 4(x) & \text { if } \alpha>0.5
\end{array}\right. \\
r_{1}(x) & =\sum_{i=1}^{m} \sum_{j=1}^{n} \sum_{k=1}^{K} C_{i j k}^{1} x_{i j k}, r_{2}(x)=\sum_{i=1}^{m} \sum_{j=1}^{n} \sum_{k=1}^{K} C_{i j k}^{2} x_{i j k}, r_{3}(x) \\
& =\sum_{i=1}^{m} \sum_{j=1}^{n} \sum_{k=1}^{K} C_{i j k}^{3} x_{i j k}, r_{4}(x)=\sum_{i=1}^{m} \sum_{j=1}^{n} \sum_{k=1}^{K} C_{i j k}^{4} x_{i j k} .
\end{aligned}
$$

Now, the safety constraint in model 4 is in the form

$$
\operatorname{Cr}\left\{\sum_{i=1}^{m} \sum_{j=1}^{n} \sum_{k=1}^{K}\left(S_{i j k}^{1}, S_{i j k}^{2}, S_{i j k}^{3}, S_{i j k}^{4}\right) x_{i j k}+(-1)\left(B_{1}, B_{2}, B_{3}, B_{4}\right) \geq 0\right\} \geq \delta
$$

Since $x_{i j k} \geq 0 x$ from Corollary 3 ('Nearest interval approximation' section), it is obvious that this constraint will be active if and only if $g \geq 0$, where

$$
g=\left\{\begin{array}{l}
(1-2 \delta)\left(\sum_{i=1}^{m} \sum_{j=1}^{n} \sum_{k=1}^{K} S_{i j k}^{1} x_{i j k}-B_{4}\right)+2 \delta\left(\sum_{i=1}^{m} \sum_{j=1}^{n} \sum_{k=1}^{K} S_{i j k}^{2} x_{i j k}-B_{3}\right) \text { if } \alpha \leq 0.5, \\
2(1-\delta)\left(\sum_{i=1}^{m} \sum_{j=1}^{n} \sum_{k=1}^{K} S_{i j k}^{3} x_{i j k}-B_{2}\right)+(2 \delta-1)\left(\sum_{i=1}^{m} \sum_{j=1}^{n} \sum_{k=1}^{K} S_{i j k}^{4} x_{i j k}-B_{1}\right) \alpha>0.5
\end{array}\right.
$$

Thus, finally an equivalent crisp form of the above model (17) can be written as 


$$
\begin{aligned}
& \operatorname{Min}\left[Z^{\prime}(x)\right] \\
& \text { s.t. } g \geq 0,
\end{aligned}
$$

with the constraints 13,14 , and 15 ,

$$
x_{i j k} \geq 0, \quad \forall i, j, k
$$

Model 5: solid transportation problem with hybrid penalties, fuzzy resources, demands, conveyance capacities, and DSM as hybrid

The respective model is formulated by assuming the unit transportation cost, desired total safety factor, and safety factor as hybrid variables:

$$
\operatorname{Min}(Z)=\sum_{i=1}^{m} \sum_{j=1}^{n} \sum_{k=1}^{K} \tilde{\hat{C}}_{i j k} x_{i j k}
$$

subject to the constraint

$$
\sum_{i=1}^{m} \sum_{j=1}^{n} \sum_{k=1}^{K} \tilde{\hat{S}}_{i j k} y_{i j k} \geq \tilde{\hat{B}}
$$

with constraints (2), (3), and (4),

$$
x_{i j k} \geq 0, \quad \forall i, j, k
$$

where $\tilde{\hat{C}}$ is the hybrid unit transportation cost from the $i$ th origin to the $j$ th destination by the $k$ th conveyance and $\tilde{\hat{B}}$ is the desired total hybrid risk factor for the whole transportation system.

\section{Solution methodology (expected value model)}

$$
\operatorname{Min} E(Z)=\sum_{i=1}^{m} \sum_{j=1}^{n} \sum_{k=1}^{K} E\left(\tilde{\hat{C}}_{i j k}\right) x_{i j k}
$$

Subject to the constraint

$$
\begin{aligned}
& \sum_{j=1}^{n} \sum_{k=1}^{k} x_{i j k} \leq E\left(\tilde{a}_{i}\right), \quad i=1,2, \ldots, m, \\
& \sum_{i=1}^{m} \sum_{k=1}^{k} x_{i j k} \geq E\left(\tilde{b}_{j}\right), \quad j=1,2, \ldots, n, \\
& \sum_{j=1}^{n} \sum_{k=1}^{k} x_{i j k} \leq E\left(\tilde{e}_{k}\right), \quad k=1,2, \ldots, K, \\
& \sum_{i=1}^{m} \sum_{j=1}^{n} \sum_{k=1}^{K} E\left(\tilde{\hat{S}}_{i j k}\right) y_{i j k} \geq E(\tilde{\hat{B}}) \\
& x_{i j k} \geq 0, \quad \forall i, j, k .
\end{aligned}
$$

\section{Numerical experiments}

Identical products are produced in three factories and sent to three warehouses for delivery through the two different types of conveyances, i.e., we considered the following $(3 \times 3 \times 2)$ solid transportation problem. 


\section{Input data}

For model 1, see Table 1 for the hybrid unit transport cost.

For model 2, see Table 2 for the crisp unit transportation cost and safety factor.

$$
\begin{aligned}
& \tilde{a}_{1}=(10,15,16,17), \tilde{a}_{2}=(14,15,18,19), \tilde{a}_{3}=(22,23,24,26), \tilde{b}_{1}=(9,10,11,12), \\
& \tilde{b}_{2}=(12,13,14,16), \\
& \tilde{b}_{3}=(5,7,8,9), \tilde{e}_{1}=(17,19,20,21), \tilde{e}_{2}=(13,14,16,17), \alpha_{i}=\beta_{j}=\gamma_{k}=0.7, \\
& F_{\alpha_{1}}=13, F_{\alpha_{2}}=14.6, \\
& F_{\alpha_{3}}=22.6, F_{\beta_{1}}=9.6, F_{\beta_{2}}=12.6, F_{\beta_{3}}=6.2, F_{\gamma_{1}}=18.2, F_{\gamma_{2}}=13.6 . \quad B=2.1 .
\end{aligned}
$$

For model 3, see Table 3 for the assumed mean and variance for random unit transportation cost and safety

$$
\begin{aligned}
& \alpha_{i}=\beta_{j}=\gamma_{k}=0.7, \text { and equal priority to mean and variance, i.e., } \lambda_{1}=\lambda_{2}=1, \\
& a_{1 c}=14.35, a_{2 c}=13.7, \\
& a_{3 c}=21.7, b_{1 c}=13.3, b_{2 c}=11.27, b_{3 c}=4.7, e_{1 c}=17.8, e_{2 c}=12.55, E(\tilde{B})=2, \\
& \quad \operatorname{Var}(\tilde{B})=2, \lambda=0.5 .
\end{aligned}
$$

\begin{tabular}{|c|c|}
\hline Unit transportation cost & Hybrid value \\
\hline$\hat{\mathrm{C}}_{111}$ & $(7,8,9)+(2,0.2)$ \\
\hline$\tilde{\hat{C}}_{211}$ & $(5,8,10)+(12,0.3)$ \\
\hline$\tilde{\hat{C}}_{311}$ & $(6,9,10)+(13,0.7)$ \\
\hline$\tilde{\hat{C}}_{121}$ & $(10,12,13)+(14,1.6)$ \\
\hline$\tilde{\hat{C}}_{221}$ & $(15,16,17)+(11,2.3)$ \\
\hline$\tilde{\hat{C}}_{321}$ & $(1,2,3)+(1,0.1)$ \\
\hline$\tilde{\hat{C}}_{131}$ & $(10,12,14)+(5,1.5)$ \\
\hline$\tilde{\hat{C}}_{231}$ & $(15,17,19)+(8,0.7)$ \\
\hline$\tilde{\hat{C}}_{331}$ & $(7,10,12)+(12,1.3)$ \\
\hline$\tilde{\hat{C}}_{112}$ & $(8,12,14)+(14,2.33)$ \\
\hline$\tilde{\hat{C}}_{212}$ & $(5,7,10)+(7,1)$ \\
\hline$\tilde{\hat{C}}_{312}$ & $(7,8,9)+(14,0.7)$ \\
\hline$\tilde{\hat{C}}_{122}$ & $(11,12,14)+(9,1.2)$ \\
\hline$\hat{\hat{C}}_{222}$ & $(17,19,21)+(19,0.9)$ \\
\hline$\hat{C}_{322}$ & $(19,20,21)+(11,1.4)$ \\
\hline$\tilde{\hat{C}}_{132}$ & $(17,18,22)+(12,1.7)$ \\
\hline$\tilde{\hat{C}}_{232}$ & $(9,13,15)+(15,0.75)$ \\
\hline$\tilde{\hat{C}}_{332}$ & $(6,10,11)+(8,1.35)$ \\
\hline
\end{tabular}

For model 4, see Tables 4 and 5 for fuzzy unit transport cost and fuzzy safety factor, respectively.

$$
\eta=0.7, \delta=0.6, \tilde{B}=(2,3,5,6)
$$

For model 5, see Table 6 for the hybrid safety factor.

$$
\tilde{\hat{B}}=(1,1.5,1.6)+(1,0.7)
$$

\section{Table 1 Hybrid unit transportation cost}


Table 2 Crisp unit transportation cost and safety factor

\begin{tabular}{llll}
\hline Unit transportation cost & Crisp value & Safety factor & Crisp value \\
\hline$C_{111}$ & 20 & $S_{111}$ & 0.25 \\
$C_{211}$ & 19 & $S_{211}$ & 0.30 \\
$C_{311}$ & 21 & $S_{311}$ & 0.82 \\
$C_{121}$ & 25 & $S_{121}$ & 0.35 \\
$C_{221}$ & 26 & $S_{221}$ & 0.30 \\
$C_{321}$ & 11 & $S_{321}$ & 0.50 \\
$C_{131}$ & 24 & $S_{131}$ & 0.80 \\
$C_{231}$ & 33 & $S_{231}$ & 1 \\
$C_{331}$ & 21 & $S_{331}$ & 0.35 \\
$C_{112}$ & 25 & $S_{112}$ & 0.42 \\
$C_{212}$ & 14 & $S_{212}$ & 0.20 \\
$C_{312}$ & 26 & $S_{312}$ & 0.20 \\
$C_{122}$ & 26 & $S_{122}$ & 0.98 \\
$C_{222}$ & 37 & $S_{222}$ & 0.82 \\
$C_{322}$ & 30 & $S_{322}$ & 0.70 \\
$C_{132}$ & 30 & $S_{132}$ & 0.50 \\
$C_{232}$ & 27 & $S_{232}$ & 0.50 \\
$C_{332}$ & 17 & $S_{332}$ & 0.20 \\
\hline
\end{tabular}

\section{Optimal results}

The optimum results of the different models using various methods are shown in Table 7.

\section{Overview of the results of the five models}

It may be noted that model 1 was solved without having to take the safety constraint, but the remaining models were solved by taking the safety constraint where safety factors were crisp, random, fuzzy, and hybrid for the respective models. After solving these five models, we have seen that the total transportation cost of model 1 is minimum compared with the other models. This is as per expectation as the nature of the four variables in safety factors for different models are different (crisp, random, fuzzy, and hybrid). Moreover, as the impreciseness for the source, demand, and conveyance capacity constraints in both models 2 and 4 are removed using credibility measure, the total satisfied demands (i.e., total transported amount) are the same, but the objective and safety values are different because of the different types of unit transportation costs (i.e., hybrid in models 1 and 5, crisp in model 2, random in model 3, and fuzzy in model 4) and safety factor (i.e., crisp in model 2, random in model 3, fuzzy in model 4, hybrid in model 5).

If we think practically the meaning of safety factor in the transportation problem, it is very large because the total transportation cost varies if the safety factor increases or decreases in some transportation problem. In this manuscript, we formulated five models with numerical example and solved these models using three methods, namely expected value modeling, chance-constrained programming, and an approach using interval approximation of fuzzy number. We have seen that if we introduce a safety factor in a transportation problem, the transportation cost for the whole transportation system increases, which was found in these five models. The transportation cost for the second, third, fourth, and fifth models were more than the transportation cost of the first model due to the safety factor. 
Table 3 Assumed mean and variance for random unit transportation cost and safety factor

\begin{tabular}{|c|c|c|c|}
\hline & & Mean & Variance \\
\hline \multirow[t]{18}{*}{ Random safety factor } & $\hat{S}_{111}$ & 0.22 & 0.11 \\
\hline & $\hat{S}_{211}$ & 0.19 & 0.1 \\
\hline & $\hat{S}_{311}$ & 0.4 & 0.2 \\
\hline & $\hat{S}_{121}$ & 0.5 & 0.14 \\
\hline & $\hat{S}_{221}$ & 0.6 & 0.2 \\
\hline & $\hat{S}_{321}$ & 0.24 & 0.3 \\
\hline & $S_{131}$ & 0.7 & 0.23 \\
\hline & $\hat{S}_{231}$ & 0.8 & 0.15 \\
\hline & $\hat{S}_{331}$ & 0.2 & 0.27 \\
\hline & $\hat{S}_{112}$ & 0.3 & 0.37 \\
\hline & $\hat{S}_{212}$ & 0.6 & 0.19 \\
\hline & $\hat{S}_{312}$ & 0.5 & 0.16 \\
\hline & $\hat{S}_{121}$ & 0.8 & 0.25 \\
\hline & $\hat{S}_{222}$ & 0.6 & 0.2 \\
\hline & $\hat{S}_{322}$ & 0.55 & 0.3 \\
\hline & $\hat{S}_{132}$ & 0.61 & 0.32 \\
\hline & $\hat{S}_{232}$ & 0.45 & 0.26 \\
\hline & $\hat{S}_{332}$ & 0.4 & 0.27 \\
\hline \multirow[t]{18}{*}{ Random unit transportation cost } & $\hat{C}_{111}$ & 19 & 2 \\
\hline & $\hat{C}_{211}$ & 19 & 1 \\
\hline & $\hat{C}_{311}$ & 17 & 0.4 \\
\hline & $\hat{C}_{121}$ & 22 & 0.75 \\
\hline & $\hat{C}_{221}$ & 22 & 0.9 \\
\hline & $\hat{C}_{321}$ & 11 & 0.5 \\
\hline & $C_{131}$ & 21 & 0.23 \\
\hline & $\hat{C}_{231}$ & 27 & 1.56 \\
\hline & $\hat{C}_{331}$ & 2 & 2.7 \\
\hline & $\hat{C}_{112}$ & 22 & 1.56 \\
\hline & $\hat{C}_{212}$ & 15 & 0.65 \\
\hline & $\hat{C}_{312}$ & 11 & 1 \\
\hline & $\hat{C}_{121}$ & 22 & 2.25 \\
\hline & $\hat{C}_{222}$ & 30 & 1.2 \\
\hline & $\hat{C}_{322}$ & 26 & 1.3 \\
\hline & $\hat{C}_{132}$ & 25 & 3.5 \\
\hline & $\hat{C}_{232}$ & 22 & 2.5 \\
\hline & $\hat{C}_{332}$ & 17 & 1.25 \\
\hline
\end{tabular}

\section{Conclusions}

To deliver the goods through different routes with different modes of conveyances is not equally safe due to insurgency and bad road. For this reason, safety constraints are introduced, and in using this concept, we have seen that the transportation cost increased when we introduced safety constraint in transportation modeling. The main objective of this paper is to present a solution procedure of a solid transportation problem under various precise and imprecise environments. In this paper, we formulated 
Table 4 Fuzzy unit transportation cost

\begin{tabular}{ll}
\hline Unit transportation cost & TrFN \\
\hline$\tilde{C}_{111}$ & $(4,5,6,11)$ \\
$\tilde{C}_{211}$ & $(7,10,11,17)$ \\
$\tilde{C}_{311}$ & $(6,8,9,10)$ \\
$\tilde{C}_{121}$ & $(5,6,8,12)$ \\
$\tilde{C}_{221}$ & $(10,11,12,14)$ \\
$\tilde{C}_{321}$ & $(12,14,17,18)$ \\
$\tilde{C}_{131}$ & $(13,14,15,20)$ \\
$\tilde{C}_{231}$ & $(15,17,19,21)$ \\
$\tilde{C}_{331}$ & $(15,17,19,21)$ \\
$\tilde{C}_{112}$ & $(3,5,7,9)$ \\
$\tilde{C}_{212}$ & $(13,15,19,20)$ \\
$\tilde{C}_{312}$ & $(11,13,14,22)$ \\
$\tilde{C}_{122}$ & $(9,10,13,14)$ \\
$\tilde{C}_{222}$ & $(12,17,18,20)$ \\
$\tilde{C}_{322}$ & $(7,8,11,12)$ \\
$\tilde{C}_{132}$ & $(6,9,13,18)$ \\
$\tilde{C}_{232}$ & $(17,18,19,21)$ \\
$\tilde{C}_{332}$ & $(18,20,21,22)$ \\
\hline
\end{tabular}

Table 5 Fuzzy safety factor

\begin{tabular}{ll}
\hline Safety factor & TrFN \\
\hline$\tilde{S}_{111}$ & $(0.18,0.22,0.25,0.3)$ \\
$\tilde{S}_{211}$ & $(0.25,0.3,0.35,0.4)$ \\
$\tilde{S}_{311}$ & $(0.39,0.44,0.49,0.55)$ \\
$\tilde{S}_{121}$ & $(0.5,0.55,0.6,0.65)$ \\
$\tilde{S}_{221}$ & $(0.65,0.7,0.75,0.8)$ \\
$\tilde{S}_{321}$ & $(0.1,0.15,0.2,0.25)$ \\
$\tilde{S}_{131}$ & $(0.73,0.78,0.83,0.88)$ \\
$\tilde{S}_{231}$ & $(0.83,0.88,0.93,0.98)$ \\
$\tilde{S}_{331}$ & $(0.25,0.3,0.35,0.4)$ \\
$\tilde{S}_{112}$ & $(0.3,0.35,0.4,0.45)$ \\
$\tilde{S}_{212}$ & $(0.72,0.77,0.82,0.87)$ \\
$\tilde{S}_{312}$ & $(0.62,0.67,0.73,0.77)$ \\
$\tilde{S}_{122}$ & $(0.84,0.89,0.94,0.99)$ \\
$\tilde{S}_{222}$ & $(0.75,0.8,0.85,0.9)$ \\
$\tilde{S}_{322}$ & $(0.65,0.7,0.75,0.8)$ \\
$\tilde{S}_{132}$ & $(0.6,0.65,0.7,0.75)$ \\
$\tilde{S}_{232}$ & $(0.50,0.55,0.6,0.65)$ \\
$\tilde{S}_{332}$ & $(0.45,0.5,0.55,0.6)$ \\
\hline
\end{tabular}


Table 6 Hybrid safety factor

\begin{tabular}{ll}
\hline Safety factor & Hybrid value \\
\hline$\hat{S}_{111}$ & $(0.17,0.18,0.19)+(0.1,0.3)$ \\
$\tilde{\hat{S}}_{211}$ & $(0.15,0.16,0.17)+(0.19,0.11)$ \\
$\hat{\hat{S}}_{311}$ & $(0.4,0.42,0.43)+(0.35,0.12)$ \\
$\tilde{\hat{S}}_{121}$ & $(0.20,0.21,0.22)+(0.25,0.14)$ \\
$\hat{\hat{S}}_{221}$ & $(0.34,0.35,0.36)+(0.35,0.2)$ \\
$\tilde{\hat{S}}_{321}$ & $(0.05,0.06,0.07)+(0.11,0.1)$ \\
$\hat{\hat{S}}_{131}$ & $(0.6,0.61,0.62)+(0.15,0.11)$ \\
$\tilde{\hat{S}}_{231}$ & $(0.7,0.71,0.73)+(0.2,0.13)$ \\
$\tilde{\hat{S}}_{331}$ & $(0.14,0.15,0.17)+(0.17,0.16)$ \\
$\tilde{\hat{S}}_{112}$ & $(0.16,0.17,0.18)+(0.19,0.18)$ \\
$\tilde{\hat{S}}_{212}$ & $(0.55,0.56,0.57)+(0.21,0.15)$ \\
$\tilde{\hat{S}}_{312}$ & $(0.2,0.21,0.22)+(0.5,0.19)$ \\
$\tilde{\hat{S}}_{122}$ & $(0.45,0.46,0.47)+(0.41,0.7)$ \\
$\tilde{\hat{S}}_{222}$ & $(0.35,0.36,0.37)+(0.44,0.17)$ \\
$\tilde{\hat{S}}_{322}$ & $(0.31,0.32,0.33)+(0.37,0.3)$ \\
$\tilde{\hat{S}}_{132}$ & $(0.39,0.4,0.41)+(0.2,0.13)$ \\
$\tilde{\hat{S}}_{232}$ & $(0.3,0.31,0.32)+(0.15,0.14)$ \\
$\tilde{\hat{S}}_{332}$ & $(0.15,0.17,0.18)+(0.31,0.2)$ \\
\hline &
\end{tabular}

Table 7 Optimum results of different models using various methods

\begin{tabular}{|c|c|c|c|c|c|}
\hline \multirow[t]{2}{*}{$\begin{array}{l}\text { Optimal } \\
\text { solution }\end{array}$} & \multicolumn{2}{|c|}{ Expected value model } & \multicolumn{2}{|c|}{$\begin{array}{c}\text { Chance-constrained } \\
\text { programming }\end{array}$} & \multirow{2}{*}{$\begin{array}{c}\text { Approach using interval approximation } \\
\text { of fuzzy number } \\
\text { Model } 3\end{array}$} \\
\hline & Model 1 & Model 5 & Model 2 & Model 4 & \\
\hline$\overline{\operatorname{Min}(Z)}$ & 267.75 & 443.06 & 456.80 & 278.50 & 326.61 \\
\hline$x_{111}$ & 6.25 & 0 & 5.60 & 0 & 0.25 \\
\hline$x_{211}$ & 0 & 0 & 0 & 0 & 0.50 \\
\hline$x_{311}$ & 0 & 0 & 0 & 4.30 & 0 \\
\hline$x_{121}$ & 0 & 0 & 0 & 7.70 & 0 \\
\hline$x_{221}$ & 0 & & & 0 & 0 \\
\hline$x_{321}$ & 11 & 11 & 12.6 & 0 & 11.27 \\
\hline$x_{131}$ & 0 & 0 & 0 & 0 & 0 \\
\hline$x_{231}$ & 0 & 0 & 0 & 0 & 0 \\
\hline$x_{331}$ & 0 & 2.50 & 0 & 6.20 & 4.7 \\
\hline$x_{112}$ & 0 & 0 & 0 & 5.30 & 0 \\
\hline$x_{212}$ & 3.25 & 9.5 & 4 & 0 & 6.82 \\
\hline$x_{312}$ & 0 & 0 & 0 & 0 & 5.73 \\
\hline$x_{122}$ & 0 & 0 & 0 & 0 & 0 \\
\hline$x_{222}$ & 0 & 0 & 0 & 0 & 0 \\
\hline$x_{322}$ & 0 & 0 & 0 & 4.90 & 0 \\
\hline$x_{132}$ & 0 & 0 & 0 & 0 & 0 \\
\hline$x_{232}$ & 0 & 0 & 0 & 0 & 0 \\
\hline$x_{332}$ & 4.75 & 2.25 & 6.20 & 0 & 0 \\
\hline
\end{tabular}


five models where penalties are crisp, random, fuzzy, and hybrid variables. Also, supplies, demands, and conveyance capacities are all fuzzy in nature, but the safety factor for models 2, 3, 4, and 5 were crisp, random, fuzzy, and hybrid variables, respectively. The present investigation is one of the few transportation models with hybrid penalties available in the literature, and for the first time, safety factors have been introduced in solid transportation problem. In this paper, we solved all mathematical problems using LINGO 12.0 software. The models could be extended to include breakable/deteriorating items, space constraints, price discount, etc. The methods, used for solutions here are quite general in nature, and these can be applied to other similar uncertain/imprecise models in other areas such as inventory control, ecology, and sustainable farm management.

\section{Author details}

1Department of Mathematics, National Institute of Technology, Agartala, Jirania 799055, West Tripura, India.

${ }^{2}$ Department of Applied Mathematics, Vidyasagar University, Midnapore 721102, West Bengal, India.

Received: 20 May 2013 Accepted: 20 November 2013

Published: 23 December 2013

\section{References}

1. Bellman, RE, Zadeh, LA: Decision-making in a fuzzy environment. Manag. Sci. 17, B141-B164 (1970)

2. Zadeh, LA: Fuzzy sets. Inf. Control 8, 338-353 (1965)

3. Zadeh, LA: Fuzzy sets as a basis for a theory of possibility. Fuzzy Set. Syst. 1, 3-28 (1978)

4. Schell, ED: Distribution of a product by several properties. Proceedings of the 2nd Symposium in Linear Programming, Washington, D.C., 27-29 January 1955, pp. 615-642. National Bureau of Standards, Washington, D.C (1955)

5. Haley, KB: The solid transportation problem. Oper. Res. 10, 448-463 (1962)

6. Kundu, P, Kar, S, Maiti, M: Multi-objective solid transportation problems with budget constraint in uncertain environment. Int. J. Syst. Sci (2013). doi:10.1080/00207721.2012.748944

7. Baidya, A, Bera, UK, Maiti, M: Multi-item interval valued solid transportation problem with safety measure under fuzzy-stochastic environment. J. Transp. Secur. 6, 151-174 (2013)

8. Baidya, A, Bera, UK, Maiti, M: Solution of multi-item interval valued solid transportation problem with safety measure using different methods. OPSEARCH, Springer (2013). doi:10.1007/s12597 013 0129-2

9. Kaufmann, A: Introduction to the Theory of Fuzzy Subsets. Academic Press, New York (1975)

10. Zimmermann, HJ: Fuzzy programming and linear programming with several objective functions. Fuzzy Set. Syst. 1, 45-55 (1978)

11. Liu, B: A survey of credibility theory. Fuzzy Optim. Decis. Making 5, 387-408 (2006)

12. Dubois, D, Prade, H: Possibility Theory: An Approach to Computerized Processing of Uncertainty. Plenum, New York (1998)

13. Chanas, S, Kuchta, D: A concept of the optimal solution of the transportation problem with fuzzy cost coefficients. Fuzzy Set. Syst. 82, 299-305 (1996)

14. Jimenez, F, Verdegay, JL: Solving fuzzy solid transportation problems by an evolutionary algorithm based parametric approach. Eur. J. Oper. Res. 117, 485-510 (1999)

15. Charnes, A, Cooper, AA: Chance-constrained programming. Manag. Sci. 6, 73-79 (1959)

16. Liu, B, Iwamura, K: Chance constrained programming with fuzzy parameters. Fuzzy Set. Syst. 94, $227-237$ (1998)

17. Liu, B, Liu, YK: Expected value of fuzzy variable and fuzzy expected value models. IEEE Trans. Fuzzy Syst. 10, 445-450 (2002)

18. Yang, L, Liu, L: Fuzzy fixed charge solid transportation problem and algorithm. Appl. Soft Comput. 7, 879-889 (2007)

19. Mula, J, Poler, R, Garcia Sabater, JP: Material requirement planning with fuzzy constraints and fuzzy coefficients. Fuzzy Set. Syst. 158, 783-793 (2007)

20. Chen, SP, Tsai, MJ: Time-cost trade-off analysis of the project network in fuzzy environment. Eur. J. Oper. Res. 212, 386-397 (2011)

21. Fegad, MR, Jadhav, VA, Muley, AA: Finding an optimal solution of transportation problem using interval and triangular membership functions. Eur. J. Sci. Res. 60, 415-421 (2011)

22. Hulsurkar, S, Biswal, MP, Sinha, SB: Fuzzy programming approach to multi-objective stochastic linear programming problem. Fuzzy Set. Syst. 88, 173-181 (1997)

23. Rao, SS: Engineering Optimization-Theory and Practice, 3rd edn. New Age International Publishers, New Delhi (2006)

24. Grzegorzewski, P: Nearest interval approximation of a fuzzy number. Fuzzy Set. Syst. 130, 321-330 (2002)

25. Liu, B: Minimax chance constrained programming model for fuzzy decision systems. Inform. Sci. 112, 25-38 (1998)

26. Yang, L, Feng, Y: A bicriteria solid transportation problem with fixed charge under stochastic environment. Appl. Math. Model. 31, 2668-2683 (2007)

27. Zhang, Q, Fan, Z, Pan, D: A ranking approach for interval numbers in uncertain multiple attribute decision making problems. Syst. Eng. - Theory Pract. 5, 129-133 (1999) 